\title{
Publisher's Note
}

Published online: 30 December 2019

(C) Springer Nature Singapore Pte Ltd. 2019

\section{Publication Schedule}

Materials Circular Economy has not accepted any manuscripts this year and will be publishing first articles online in 2020. 